P86 (continued)

recruited from the WIC program at a community health center.

Outcome Measures and Analysis: The knowledge section of the self-reported questionnaire consisted of three scales (32 items) that assessed general knowledge of anemia, knowledge of anemia during pregnancy, and knowledge of iron rich food sources. Participants' demographic data was collected, and blood hemoglobin levels were recorded. Descriptive and inferential statistics were used to analyze data. Principal Component Analysis was used to validate the three knowledge scales, and to transform participants' scores into tertiles, namely poor, adequate and good. Inter-item reliability was evaluated using the Cronbach's $\alpha$.

Results: Seventy-eight percent of participants had normal blood hemoglobin levels, while $22 \%$ were anemic. Although $83 \%$ of participants had adequate knowledge (responded correctly to $67 \%$ of the questions) of anemia, anemia during pregnancy, and iron rich food sources, only $5 \%$ had good knowledge of these factors.

Conclusions and Implications: The $22 \%$ prevalence of anemia in our sample of pregnant women is higher than the $17 \%$ reported nationally. These results suggest that pregnant women participating in the WIC program can benefit from more targeted nutrition education on anemia prevention and iron rich food sources.

Funding: Western Michigan University's Department of Family and Consumer Sciences Toms Research Grant

\section{P87 Longitudinal Evaluation of Fruit and Vegetable Preferences Among K-2nd Grade Students Participating in the Fresh Fruit and Vegetable Program (FFVP)}

Natalie Masis, MS, masis2@illinois.edu, University of Illinois at Urbana-Champaign, 238 Bevier Hall, 905 South Goodwin Avenue, Urbana, IL 61801;

Jennifer McCaffrey, PhD, MPH, RD, University of Illinois Extension; Susan L. Johnson, PhD, University of Colorado at Denver; Karen Chapman-Novakofski, PhD, LDN, RD, University of Illinois

Objective: To evaluate fruit and vegetable preferences over time, with repeated experience, as part of the Fresh Fruit and Vegetable Program (FFVP).

Study Design, Setting, Participants, Intervention: Fruits $(\mathrm{F}=28)$ and vegetables $(\mathrm{V}=29)$ were distributed twice a week, over 35 weeks, at a participating FFVP-school $(\mathrm{n}=$ 236 students, 12 teachers, K-2nd grade). A 3-point Likert emoticon scale was used to evaluate student FV preferences.

Outcome Measures and Analysis: Preference ratings were analyzed, over 35 weeks, by descriptive statistics, Spearman's rho correlations, Mann-Whitney U, and linear regression with predictor variables including grade level, fruit or vegetable served, and time (week).
Results: For the 57 different FV rated for preference, ratings $(n=10,335, F=5,121 ; V=5,214)$ revealed that $F$ had higher frequency of children choosing "I like it" than for V (78\% F; 38.2\% V). Preferences for F were higher ranked than for $\mathrm{V}(\mathrm{p}<0.01)$ and liking distribution (liked, okay, didn't like) was different between $\mathrm{F}$ and $\mathrm{V}(\mathrm{p}<.001)$. Significant relations were found between liking and: grade $(\mathrm{r}=-0.02, \mathrm{P}=0.02)$, and time $(\mathrm{r}=-0.09, \mathrm{p}<0.001)$. Models indicated that V served (compared to fruits; $\beta=-$ $.40)$, time point $(\beta=-.07)$, and grade level $(\beta=-.02)$ accounted for significant variance for preference ratings $(\mathrm{R} 2=0.17, \mathrm{p}<.001)$, indicating that preference ratings went down over time. When modeled independently, this remained true for $\mathrm{V}(\mathrm{R} 2=0.007, \mathrm{p}<0.001)$, but only time remained significant in the $\mathrm{F}$ preference model $(\mathrm{R} 2=0.008, \mathrm{p}<0.001)$.

Conclusions and Implications: Fruits were preferred over vegetables. Overall preference ratings were negatively impacted by time, grade level, and vegetables served. Being exposed to a variety of FV, generally, did not improve ratings for vegetables.

Funding: University of Illinois Extension

\section{P88 Maryland Expanded Food and Nutrition Education Program Participants Show Improved Diet Quality as Determined by HEI Scores}

Kavitha Sankavaram,PhD, kavitha@umd.edu, University of Maryland, College Park, 0102 Skinner Building, 4300 Chapel Lane, University of Maryland, College Park, MD 20742; Mira Mehta, PhD, University of Maryland, College Park

Objective: To evaluate the effectiveness of Expanded Food and Nutrition Education Program (EFNEP) by comparing participants' overall diet quality using Healthy Eating Index Score (HEI) when entering and exiting the program. Target audience were limited income families of various races/ethnicities: Caucasian, Black or African American, Asian, Multiple Races, Hispanic/ Latinos.

Theory, Prior Research, Rationale: This study used the socioecological model as its theoretical foundation. Evidence suggests that an individual's level of nutrition knowledge has important influence on their HEI Score.

Description: HEI provides an overall picture of the types and quantity of foods people eat, their compliance with specific dietary recommendations, and the variety in their diets. Other factors explaining variations in HEI's across individuals are income and education levels, race, ethnicity, and age. In this study, pre- and post-test data collected from participants of various race/ethnicities were compared.

Evaluation: Secondary analyses were performed on data collected from 2013-2016. Dietary recalls were collected by paraprofessionals. Outcome measures were the differences between HEI-2005 scores at entry and exit. Significance between each group was 
P88 (continued)

determined using the Wilcoxon signed-rank test and differences among all groups by Kruskal Wallis test. Out of the total participants $(\mathrm{n}=4457)$ reported, $32 \%$ were Caucasian, 27\% Black, 1\% Asian, 2\% multiple races, and $38 \%$ of Latino ethnicity. The mean change in HEI-2005 score from entry to exit was 8.2 points in Caucasian, 8.1 in Black, 3.8 in Asian, 9.2 in multiple races, and 9.6 in Latino participants $(\mathrm{p}<0.01)$.

Conclusions and Implications: Overall, the average change in HEI scores increased by 7.8 points indicating that nutrition knowledge likely improved and led to short-term improvement in diet quality.

Funding: USDA

\section{P89 Multifaceted Intervention Increases WIC Farmers Market Nutrition Program Check Redemption and Suggests Policy Changes}

Miriam Seidel, MS, RD, mseidel@chatham.edu, Chatham University, 6035 Ridge Road, Gibsonia, PA 15044; Malik Hamilton, BS, Chatham University; Leslie Gordon, BA; Callie Carioti, BS

Objective: Increase WIC Farmers Market Nutrition Program (FMNP) check redemption rate. Target audience was WIC participants in a low income $47.6 \%$ white, 46.9\% African American neighborhood adjacent to the city of Pittsburgh.

Theory, Prior Research, Rationale: The Special Supplemental Nutrition Program for Women, Infants and Children (WIC) FMNP aims to increase consumption of fresh fruits and vegetables for WIC families while supporting local farmers who sell at area farmers markets (FM). Yet, FMNP check utilization in PA is just over 50\% and is lower in the targeted neighborhood. Barriers as well as aids to FMNP utilization and produce consumption are well documented and informed the intervention, CRUNCH!: Eat Fresh, Eat Healthy, Move More. A review of FMNP procedures in several states is informing policy recommendations for PA.

Description: Using a quasi-experimental research design, the June through early November intervention included cooking demonstrations in the WIC clinic; informal tours of FMs; and community garden visits.

Evaluation: State data provided check redemption rates. Baseline and follow-up questionnaires explored barriers to FMNP check redemption, aids to fruit and vegetable consumption and pre- and post-intervention consumption data. Participation in the intervention activities were tracked.

Conclusions and Implications: The intervention significantly $(\mathrm{p}<.001)$ increased the FMNP check redemption rate by program participants (46\%) compared to nonparticipants (39\%). Participation in the intervention activities was lower than expected. Barriers were consistent with the literature and there was no change in fruit or vegetable consumption. Program findings led to discussion of local issues and state policies regarding interpretation of federal
FMNP regulations. A local stakeholder group is currently exploring policy recommendations.

Funding: Aetna Foundation

\section{P90 "Where are the Tomatoes From?" Restaurant Wait Staff Ability to Address Customer Questions about Food Sources}

Laura Dragon, MS, 1rdragon@syr.edu, Syracuse University, 150 Crouse Drive, Syracuse, NY 13244;

Jennifer Wilkins, PhD, RD, Syracuse University

Objective: The dramatic increase in local food demand has been a leading consumer trend over the past few decades. Consumers have sought local food at farmers' markets, roadside stands and grocery stores and on restaurant menus. Increasingly, waiters, as the primary customer contact, are called upon to provide information about sources of menu items and ingredients. This pilot study explored waiter understanding of, and training related to, local foods (LF), characteristics of customer inquiries, LF sources and wait staff confidence in addressing customer questions about LF.

Study Design, Setting, Participants: Local food promoting restaurants (LFPRs), where food sourcing is featured in establishment marketing, were included in this study. Nineteen waiters employed at eight LFPR in one central New York urban county were surveyed.

Outcome Measures and Analysis: Quantitative data were analyzed primarily through descriptive statistics. Mann-Whitney U-Test was used to determine whether training or confidence differed among waiters that were accurate or inaccurate in identifying the restaurant's ingredient sources.

Results: Ten waiters (63\%) had at least a bachelor's degree; fourteen $(82 \%)$ were white, and ages ranged from 22 to 46 . Nearly two-thirds of waiters were "very confident" in addressing customers' LF questions. Less than one-third of waiters accurately identified sources of menu ingredients. No significance was found between accuracy and confidence level in addressing LF questions.

Conclusions and Implications: These preliminary data suggest waiters may have a false sense of confidence in addressing LF questions indicating a need for more training on local foods. Further, waiter claims about food sources in LFPRs need to be verified with procurement records.

Funding: None

\section{P91 Back to the Future: National Food Conservation Recommendations from 1917-18 \\ Janet Schwartz, MS, RD, jschwartz@framingham.edu, Framingham State University, 100 State Street, Framingham, MA 01701}

Objective: To investigate and describe an historical national food conservation effort and celebrate the 100th anniversary of the US Food Administration 